\section{Comment on: Concerns of using sialidase fusion protein as an experimental drug to combat seasonal and pandemic influenza}

John M. Nicholls ${ }^{1} \dagger$, Laura M. Aschenbrenner ${ }^{2} \dagger$, James C. Paulson ${ }^{3}$, Erin R. Campbell ${ }^{2}$, Michael P. Malakhov², David F. Wurtman ${ }^{2}$, Mang $\mathrm{Yu}^{2}$ and Fang Fang ${ }^{2 *}$

${ }^{1}$ Pathology Department, The University of Hong Kong, Pok Fu Lam, Hong Kong, Hong Kong SAR; ${ }^{2}$ NexBio Inc., 10665 Sorrento Valley Road, San Diego, CA 92121, USA; ${ }^{3}$ Departments of Chemical Physiology and Molecular Biology, The Scripps Research Institute, 10550 N. Torrey Pines Road, La Jolla, CA 92037, USA

*Corresponding author. Tel: +1-858-452-2631; Fax: + 1-858452-0133; E-mail: ffang@ nexbio.com

$\uparrow$ These authors contributed equally to this correspondence.

Sir,

Zhang $^{1}$ questioned the validity of developing a sialidase fusion protein (DAS181) as an experimental drug against influenza. He raised two main concerns-first, that sialidase treatment would result in a greater chance of bacterial infection by exposing novel cryptic binding sites to bacterial pathogens and second, that influenza virus (IFV) receptors may be different from sialic acids (SA); thus, sialidase treatment may be ineffective. Although we agree with Zhang that the safety and efficacy of any novel therapeutic candidate, including DAS181, needs to be evaluated cautiously, statements made by him on sialidase treatment and the general view presented on the role of SA in IFV infection are either incomplete or incorrect. We thus offer the following corrections.

It is known that IFV infection is associated with an increased risk of secondary infection by Streptococcus pneumoniae. ${ }^{2}$ A clear distinction that needs to be made, however, is whether the secondary S. pneumoniae infection arises from viral neuraminidase (a sialidase) by itself or from the secondary effects of IFV infection. Scientific evidence clearly supports the latter, as influenza infection in the trachea and bronchus causes much deeper changes to the airway epithelium than could be caused by treatment of the epithelial cell surface with a sialidase. Influenza infection in humans results in extensive denudation of the ciliated epithelial cells down to the basal cells, or even to the basement membrane. ${ }^{3}$ Similar changes are also present in mice infected with IFV. ${ }^{4}$ When $S$. pneumoniae infection does occur, it is seen at the exposed layers of basal cells or basement membrane, but not on the intact epithelial surface. ${ }^{4}$ Thus, contrary to Zhang's concern, the data from Plotkowski et al ${ }^{4}$ provide direct evidence that increased $S$. pneumoniae infection is the result of airway epithelial damage caused by IFV infection. By inhibiting IFV infection as the primary cause of the epithelial damage, sialidase treatment can protect the airway epithelium from inflammation and denudation and thus can potentially prevent secondary bacterial infection.

Zhang suggested that sialidase increases S. pneumoniae adherence by exposing cryptic receptors. SA is usually connected via $\alpha 2-6$ or $\alpha 2-3$ linkage to either Gal $\beta 1-4 G l c N A c$ or Galß1-3GalNAc. Thus, the inner polysaccharide or 'cryptic' fragments exposed after sialidase treatment would be predominantly Galß1-4GlcNAc and Galß1-3GalNAc. Using an established methodology, we stained normal and DAS181-treated human tracheal tissue sections with lectins. We observed that DAS181 treatment abolished SA binding by Sambucus nigra agglutinin (SNA) (which binds SA $\alpha 2-6$ ). Importantly, Gal $\beta 1-4 G l c N A c$ [bound by Datura stramonium agglutinin (DSA)] was abundantly present on the ciliated surface of tracheal epithelium in the native state as well as following DAS181 treatment (Figure 1). Consistent with our observation, the matrix-assisted laser desorption ionization mass spectrometry (MALDI-MS) glycan profile analysis of the human bronchial epithelium also revealed abundant Galß1-4GlcNAc structures in the absence of sialidase. ${ }^{5}$ Galß1-3GalNAc expression was low and restricted to goblet cells and basal cells in the control tissue; it was modestly increased only in the goblet cells after DAS181 treatment (Figure 1). Noticeably, mass spectrometric analysis has also revealed numerous Galß1-4GlcNAc structures in the normal lung tissue (http:// www.functionalglycomics.org/glycomics/publicdata/glycoprofiling. jsp?species=Human). Together, these studies demonstrate that the inner polysaccharide structures exposed by desialylation already exist on the normal respiratory surface. As a result, sialidase treatment is unlikely to introduce de novo bacterial adhesion sites.

We conducted further studies to evaluate whether sialidase treatment would increase colonization of respiratory epithelial cells by $S$. pneumoniae, Haemophilus influenzae or Pseudomonas aeruginosa. DAS181 treatment of A549 cells did not increase cell adhesion by these bacteria over a broad range of bacterial inputs (L. M. Aschenbrenner and F. Fang, unpublished results). In the well-differentiated human airway epithelium (HAE) cultures, DAS181 treatment did not increase adhesion of $S$. pneumoniae (Figure 2) and did not result in any cytopathic effect. Together, our findings support previous observations, indicating that removing SA alone does not have a significant impact on bacterial cell adhesion.

Zhang also raised concerns over the entry of IFV into desialylated cells by citing isolated reports that investigate aspects of IFV entry in cell culture models. Evidence accumulated over the past six decades has indisputably demonstrated the key role of SA as the IFV receptor. ${ }^{6,7}$ The purpose of these cited reports was to investigate other aspects of IFV entry. None of them disputed the role of SA in promoting IFV infection. For example, although Stray et $a l .{ }^{8}$ demonstrated non-SA-dependent entry of IFV into cells at high doses, they also demonstrated $90 \%$ to $99.999 \%$ reduction of IFV multicycle infectivity in desialylated MadinDarby canine kidney (MDCK) cells. Chu and Whittaker 9 showed that glycoproteins which contain sialylated $\mathrm{N}$-linked glycans were critical for productive entry of IFV into cells. These results in fact support the role of sialylated glycans as the receptors of IFV.

DAS181 (Fludase ${ }^{\mathrm{TM}}$ ) is being developed as a potential broad-spectrum prophylactic and therapeutic agent for seasonal 


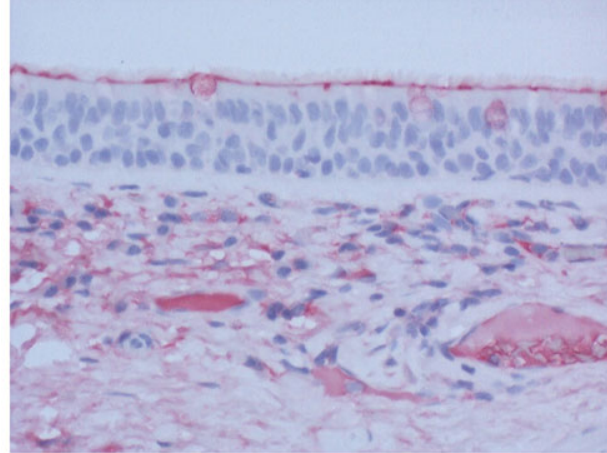

Untreated SNA

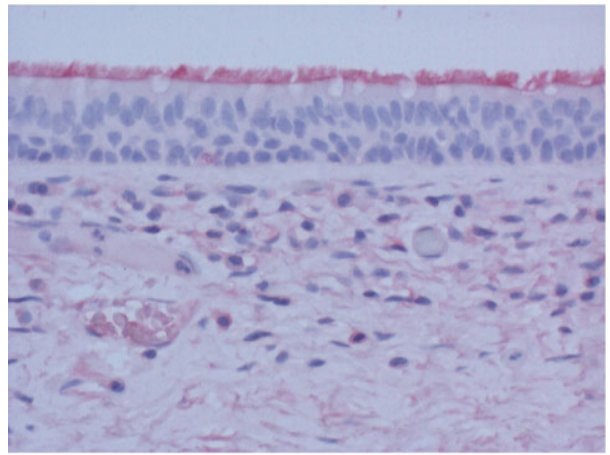

Untreated DSA

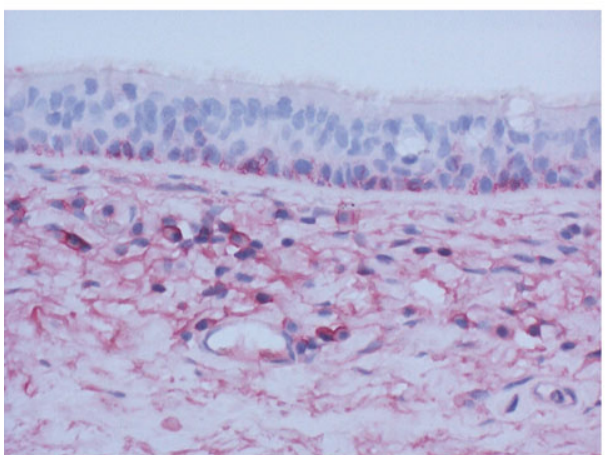

Untreated PNA

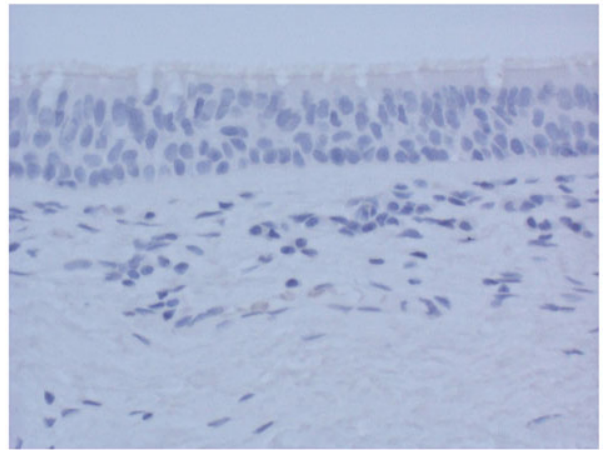

DAS181-treated SNA

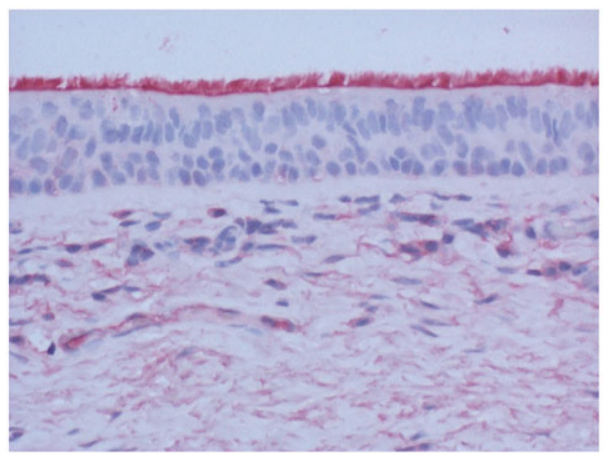

DAS181-treated DSA

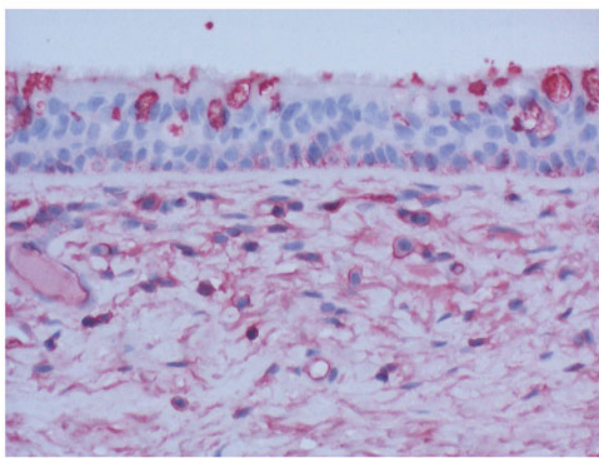

DAS181-treated PNA

Figure 1. SNA, DSA and peanut (Arachis hypogaea) agglutinin (PNA) binding to human tracheal tissue sections. Lectin-binding studies of fixed normal human bronchial mucosal sections following $2 \mathrm{~h}$ of incubation with control PBS (untreated) or $500 \mathrm{U} / \mathrm{mL}$ of DAS181 at $37^{\circ} \mathrm{C}$ (DAS181-treated) and then stained with digoxigenin-labelled SNA, DSA and PNA (Roche Dig Glycan Detection Kit). Colour development was with New Fuchsin Alkaline Phosphatase Substrate Solution and counterstained with haematoxylin. All magnifications $\times 200$.

influenza-like illness and for pandemic influenza. We have reported results that demonstrate potent in vitro and in vivo activities of DAS181 against a broad panel of IFV strains, including the highly pathogenic H5N1 strain. ${ }^{10,11}$ In order to fully address the legitimate questions on drug safety, in the ongoing Phase 1 clinical trial, we are closely monitoring the status of bacterial colonization among an extensive panel of safety parameters.

Preparing the world to safeguard itself against an influenza pandemic poses daunting challenges to the scientific community and industry alike. We genuinely welcome constructive scientific input from our colleagues and focus our effort on the important task in front of us.

\section{Acknowledgements}

This study was supported by funding from the Research Grants Council, Hong Kong SAR, and NIH grants 2R44AI056786 and 1U01AI070281.

\section{Transparency declarations}

L. M. A., J. C. P., E. R. C., M. P. M, D. F. W., M. Y. and F. F. have shares in NexBio Inc., which is the sponsor of DAS181 development. J. M. N.: none to declare. 
Letters to the Editor

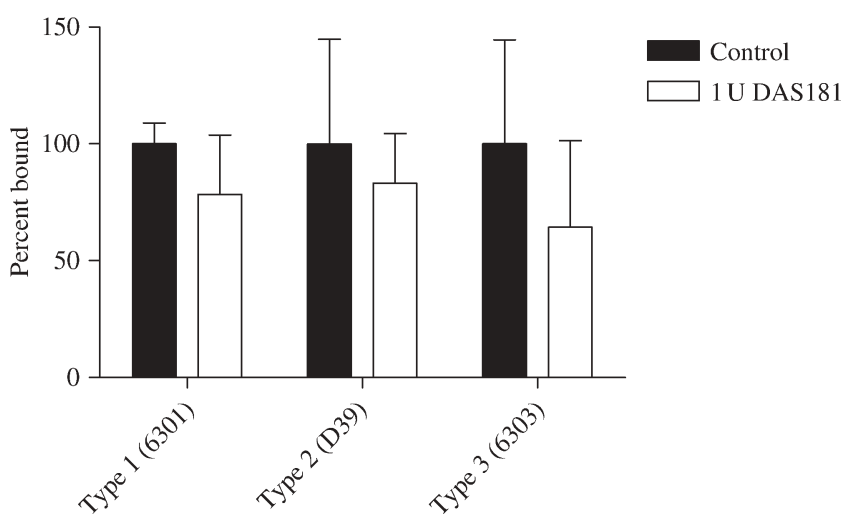

Figure 2. S. pneumoniae adhesion to DAS181-treated HAE. HAE cultures grown in a 96-well format (Mattek Corp.) were treated with either DMEMF12 with $4 \%$ BSA or 1 U/well DAS181 on the apical surface for $2 \mathrm{~h}$ at $37^{\circ} \mathrm{C}$. The surface SA level was reduced by $80 \%$ after treatment (data not shown). After washing, the cells were incubated with the following S. pneumoniae strains: $6 \times 10^{6} \mathrm{cfu} /$ well Type 1 (6301), $6 \times 10^{6} \mathrm{cfu} /$ well Type 2 (D39) and $4 \times 10^{4} \mathrm{cfu} /$ well Type 3 (6303). After $1 \mathrm{~h}$ incubation and washing, the adherent bacteria and cells were removed from the plate by trypsinization and quantified by the viable plate count assay. Colonies were counted. Samples showing bacterial adhesion to the cultures treated with medium alone were controls that were normalized as $100 \%$ bound. Error bars represent one standard deviation above the mean of triplicate samples. Statistical significance between the control and DAS181-treated sample was tested with Student's $t$-test. No statistically significant differences were detected.

\section{References}

1. Zhang $\mathrm{H}$. Concerns of using sialidase fusion protein as an experimental drug to combat seasonal and pandemic influenza. $J$ Antimicrob Chemother 2008; 62: 219-23.

2. van der Sluijs KF, van Elden LJ, Nijhuis $M$ et al. Involvement of the platelet-activating factor receptor in host defense against Streptococcus pneumoniae during postinfluenza pneumonia. Am J Physiol Lung Cell Mol Physiol 2006; 290: L194-9.

3. Walsh JJ, Dietlein LF, Low FN et al. Bronchotracheal response in human influenza. Type A, Asian strain, as studied by light and electron microscopic examination of bronchoscopic biopsies. Arch Intern Med 1961; 108: 376-88.

4. Plotkowski MC, Puchelle E, Beck G et al. Adherence of type I Streptococcus pneumoniae to tracheal epithelium of mice infected with influenza A/PR8 virus. Am Rev Respir Dis 1986; 134: 1040-4.

5. Chandrasekaran A, Srinivasan A, Raman R et al. Glycan topology determines human adaptation of avian H5N1 virus hemagglutinin. Nat Biotechnol 2008; 26: 107-13.

6. Paulson JC. In: Conn M, ed. Interactions of Animal Viruses with Cell Surface Receptors. New York, NY: Academic Press, 1985; 131-219.

7. Skehel JJ, Wiley DC. Receptor binding and membrane fusion in virus entry: the influenza hemagglutinin. Annu Rev Biochem 2000; 69: $531-69$.

8. Stray SJ, Cummings RD, Air GM. Influenza virus infection of desialylated cells. Glycobiology 2000; 10: 649-58.

9. Chu VC, Whittaker GR. Influenza virus entry and infection require host cell N-linked glycoprotein. Proc Natl Acad Sci USA 2004; 101: 18153-8.

10. Malakhov MP, Aschenbrenner LM, Smee DF et al. Sialidase fusion protein as a novel broad-spectrum inhibitor of influenza virus infection. Antimicrob Agents Chemother 2006; 50: 1470-9.

11. Belser JA, Lu X, Szretter KJ et al. DAS181, a novel sialidase fusion protein, protects mice from lethal avian influenza H5N1 virus infection. J Infect Dis 2007; 196: 1493-9.

Journal of Antimicrobial Chemotherapy

doi:10.1093/jac/dkn220

Advance Access publication 10 June 2008

\section{Concerns of using sialidase fusion protein as an experimental drug to combat seasonal and pandemic influenza-author's response}

\author{
Hong Zhang* \\ Z-BioMed Inc., 15725 Crabbs Branch Way, Rockville, \\ MD 20855, USA
}

Keywords: neuraminidase, pathogenic factor, S. pneumoniae

*Tel: +1-301-258-8968; Fax: +1-301-260-0622;

E-mail: hzhang@zbiomed.com

Sir,

In my Leading article, I discussed 12 papers that studied bacterial infection. ${ }^{1}$ In their commentary on my article, Nicholls $e t \mathrm{al}^{2}$ chose to address only one of them. Studies by several groups using different animal models have demonstrated that influenza virus infection contributes significantly to secondary bacterial pneumonia and that neuraminidase activity in influenza viruses is a predictor of mortality from secondary bacterial pneumonia. ${ }^{3-6}$ Peltola et $a l .^{4}$ concluded in one of their papers 'This action of NA also promotes adherence and invasion of Streptococcus pneumoniae, because cleavage of sialic acid from the surface of host cells exposes cryptic receptors for $S$. pneumoniae. Bacteria that can successfully invade the lower respiratory tract typically express NA for this purpose'.

The fact that influenza A viruses, including recent $\mathrm{H} 3 \mathrm{~N} 2$ clinical isolates, can infect desialylated cells clearly demonstrates that sialic acid-independent entry can occur, ${ }^{7}$ but this should not be misconstrued as 'influenza virus (IFV) receptors may be different from sialic acids (SA)'.

Nicholls et $a l^{2}$ provided data on sialidase treatment and bacterial adherence to address some of the concerns I raised. However, I have some specific concerns regarding this. Figure 1 in their comment has no quantification and there is a clear increase of DSA and PNA binding after DAS181 treatment. To describe each of these as a 'modest' increase is subjective and debatable; indeed, the MALDI-MS data are not quantitative and the relative amounts were not disclosed. The statement by Nicholls et al. ${ }^{2}$ that DAS181 treatment did not affect S. pneumoniae binding 'over a broad range of bacterial input' is inaccurate as their Figure 2 shows that at lower bacterial doses there was an apparent increase $(P<0.05)$.

I believe that the concerns raised in my original article remain and warrant further consideration and investigation to determine their ramifications. 